\title{
SMOOTH SOLUTIONS OF INITIAL-VALUE PROBLEMS IN REGIONS WITH CORNERS
}

\author{
E. C. SVENDSEN
}

\begin{abstract}
The method of images is used to show the existence and uniqueness of smooth solutions of initial-value problems in regions with corners.
\end{abstract}

1. Introduction. Corners may destroy the existence or uniqueness of smooth solutions of initial-value problems for partial differential equations $[\mathbf{3}, \mathbf{4}]$. This paper is about initial-value problems in which corners do not cause such trouble. The method of images is used. This method is applicable to many fundamental domains for group actions, some of which Keller [1] discussed. Here the method is applied to products of intervals, which are regarded as fundamental domains for groups of isometries of $R^{n}$. Functions will be vector-valued, and so the boundary conditions for different faces of the domain must be compatible (as they automatically were for Keller because his functions were scalar-valued). The boundary conditions must also be compatible with the differential equation. In this paper we formulate compatibility conditions, and then show that they imply the existence and uniqueness of smooth solutions.

2. The main result. The infinitesimal generator of time evolution will be a linear differential operator $A$ acting on functions from $R^{n}$ to $C^{m}$. We suppose that $A$ is translation-invariant and formally skew-adjoint.

The spatial region will be $\Omega=\Omega_{1} \times \cdots \times \Omega_{n}$, where $\Omega_{i}$ is the closure of the interval $\left(a_{i-}, a_{i+}\right)$. The $a_{i \sigma}$ may be infinite; let $S$ be the set of $(i, \sigma)$ for which $a_{i \sigma}$ is finite. Boundary conditions will be specified in terms of subspaces $V_{i \sigma}$ of $C^{m}$, one for each $(i, \sigma)$ in $S$. For convenience, let $V_{i \sigma}^{j}$ be $V_{i \sigma}$ if $j$ is even and $V_{i \sigma}^{\perp}$ if $j$ is odd.

We consider the initial-value problem

$$
\partial \tilde{f} / \partial t=A \tilde{f}, \quad \tilde{f}(, t)=f
$$

on $\Omega \times R$, with $f$ and each $\tilde{f}(, t)$ satisfying the boundary conditions

$$
D_{i}^{j} f \in V_{i \sigma}^{j}
$$

on the intersections $(\partial \Omega)_{i \sigma}$ of $\partial \Omega$ and the hyperplanes $x_{i}=a_{i \sigma}$.

As usual, $H^{\infty}\left(\Omega, C^{m}\right)$ denotes the Sobolev space of functions $f$ in $C^{\infty}\left(\Omega, C^{m}\right)$ such that $f$ and its derivatives (of all orders) are square-integrable. We use a smaller space, $H_{V}^{\infty}\left(\Omega, C^{m}\right)$, the set of $f$ in $H^{\infty}\left(\Omega, C^{m}\right)$ satisfying condition $(2.2)$ on $(\partial \Omega)_{i \sigma}$ for all $j$ in $\{0,1, \ldots\}$ and $(i, \sigma)$ in $S$.

Received by the editors June 9, 1983.

1980 Mathematics Subject Classification. Primary 35B65; Secondary 35L50.

(c) 1984 American Mathematical Society $0002-9939 / 84 \$ 1.00+\$ .25$ per page 
Here is our main result:

THEOREM 1. Suppose that (H1) the orthogonal projections $P_{i \sigma}$ onto the $V_{i \sigma}$ commute, and that (H2) $A$ maps $H_{V}^{\infty}\left(\Omega, C^{m}\right)$ to itself. Then for each $f$ in $H_{V}^{\infty}\left(\Omega, C^{m}\right)$, there is a unique $\tilde{f}$ in $C^{\infty}\left(\Omega \times R, C^{m}\right)$ such that $\tilde{f}$ solves problem (2.1) and each $\tilde{f}(, t)$ is in $H_{V}^{\infty}\left(\Omega, C^{m}\right)$.

Let $A=\sum A^{\alpha} D^{\alpha}$. Hypothesis (H1) holds if and only if

$$
A^{\alpha} V_{i \sigma}^{j} \subset V_{i \sigma}^{j+\alpha_{i}}
$$

for all $\alpha, j$, and $(i, \sigma)$.

Massey and Rauch, in their study [2] of problem (2.1) with $A$ a first-order operator and $\Omega$ an open subset of $R^{n}$ having a smooth boundary, imposed conditions like (2.3) and obtained a result like ours.

3. Examples. Schrödinger's equation with Dirichlet or Neumann boundary conditions provides examples of Theorem 1 . (Here $m$ is $1, A$ is $i \Delta$, and each $V_{i \sigma}$ is $\{0\}$ (Dirichlet) or $C$ (Neumann).) Other examples are provided by Maxwell's equations with the boundary conditions for a perfect conductor. (Here $n$ is 3, $m$ is 6 ,

$$
A=\left[\begin{array}{cc}
0 & I_{3} \\
-I_{3} & 0
\end{array}\right] \nabla x
$$

and each $V_{i}$ is the set of $(E, B)$ with $E$ perpendicular to $(\partial \Omega)_{i \sigma}$ and $B$ parallel to it.)

The hypotheses of Theorem 1 cannot be eliminated, as these two examples on $\Omega=[0, \infty)^{2}$ show: In the first example,

$$
A=\left[\begin{array}{cc}
1 & 0 \\
0 & -1
\end{array}\right] \nabla \cdot(1,1)
$$

and $V_{1-}=V_{2-}$ is the set of $\left(f_{1}, f_{2}\right)$ in $C^{2}$ with $f_{1}=f_{2}$. Then (H1) holds, but (H2) and the conclusion do not. In the second example,

$$
A=\left[\begin{array}{cc}
M \nabla \cdot(1,1) & 0 \\
0 & M \nabla \cdot(1,-1)
\end{array}\right],
$$

where

$$
M=\left[\begin{array}{cc}
I_{2} & 0 \\
0 & -I_{2}
\end{array}\right] .
$$

Let $U_{31}, U_{41}, U_{23}$, and $U_{24}$ be $2 \times 2$ unitary matrices. For each $f$ in $C^{8}$, write $f=\left(f_{1}, f_{2}, f_{3}, f_{4}\right)$, the $f_{i}$ being in $C^{2}$. Then $V_{1-}$ is the set of $f$ in $C^{8}$ with $f_{4}=U_{41} f_{1}$ and $f_{2}=U_{23} f_{3}$, and $V_{2-}$ is the set of $f$ in $C^{8}$ with $f_{3}=U_{31} f_{1}$ and $f_{2}=U_{24} f_{4}$. Suppose that the $U$ 's are square roots of the identity matrix $I_{2}$ and that $U_{23} U_{31} \neq U_{24} U_{41}$. Then (H2) holds, but (H1) and the conclusion do not. In these two examples, waves lose differentiability along the line $x_{1}=x_{2}$ during reflection from the boundary. (In the second example they even lose continuity.) The corner is responsible; indeed, in the half-space problems associated with these problems, both (H1) and (H2) hold, and her.ce so does the conclusion. (Osher [3] and Sarason and Smoller [4] gave examples of even worse behavior at corners.) 
4. Proof of Theorem 1. In this section we suppose the projections $P_{i \sigma}$ commute.

Here is a thumbnail sketch of our construction of $\tilde{f}: \Omega$ is a fundamental domain for a group $G$. The $V_{i \sigma}$ determine a unitary representation of $G$ on $C^{m}$. Each function $f$ on $\Omega$ has an equivariant extension $E f$ to $R^{n}$, which is invariant under a group $T$ of translations. Associated with $T$ is a Fourier transform operator $F$, under which $A$ becomes a multiplication operator $\hat{A}$. The solution of problem (2.1) is given by

$$
\tilde{f}(, t)=E^{-1} F^{-1} e^{\hat{A} t} F E f .
$$

$G$ is the group of isometries of $R^{n}$ generated by the reflections $R_{i \sigma}$ across the hyperplanes $x_{i}=a_{i \sigma}$ (for $(i, \sigma)$ in $S$ ). We will work with the unitary operators $U_{i \sigma}=2 P_{i \sigma}-I$. Since the $P_{i \sigma}$ commute, so do the $U_{i \sigma}$. Therefore there is a unique unitary representation $U$ of $G$ on $C^{m}$ such that $U\left(R_{i \sigma}\right)=U_{i \sigma}$ for all $(i, \sigma)$ in $S$. Let $H_{U}^{\infty}\left(R^{n}, C^{m}\right)$ be the space of functions in $C^{\infty}\left(R^{n}, C^{m}\right)$ that are equivariant with respect to $U$ (that is, satisfy $f(g x)=U(g) f(x)$ for all $g$ and $x$ ) and are extensions of functions in $H^{\infty}\left(\Omega, C^{m}\right)$.

LEMMA 1. (1) There is a unique bijection $E$ from $H_{V}^{\infty}\left(\Omega, C^{m}\right)$ to $H_{U}^{\infty}\left(R^{n}, C^{m}\right)$ such that $E f$ is an extension of $f$ for all $f$ in $H_{V}^{\infty}\left(\Omega, C^{m}\right)$. (2) $A$ maps $H_{V}^{\infty}\left(\Omega, C^{m}\right)$ to itself if and only if $A$ maps $H_{U}^{\infty}\left(R^{n}, C^{m}\right)$ to itself. (3) If $A$ is as in part (2), then $E A=A E$ on $H_{V}^{\infty}\left(\Omega, C^{m}\right)$.

Before proving Lemma 1 , we remark that $A$ maps $H_{U}^{\infty}\left(R^{n}, C^{m}\right)$ to itself if and only if

$$
A^{\alpha} U(g)=\sigma(\alpha, g) U(g) A^{\alpha}
$$

for all $\alpha$ and $g$, where $\sigma(\alpha, g)= \pm 1$ is defined as follows: Since $g$ is an isometry of $R^{n}$, there is a unique orthogonal transformation $O(g)$ such that $g x=O(g) x+g 0$ for all $x$. More specifically, $O(g)$ is an element of the group generated by the reflections across the coordinate hyperplanes of $R^{n}$, and so $O(g) e_{i}=\sigma_{i}(g) e_{i}$ for some $\sigma_{i}(g)$ in $\{-1,1\}$. Then

$$
\sigma(\alpha, g)=\prod_{i=1}^{n} \sigma_{i}(g)^{\alpha_{i}}
$$

Conditions (4.1) are equivalent to conditions (2.3) (by part (2) of Lemma 1).

PROOF OF LEMMA 1. (1) If there is such an $E$, it must be given by $E f(x)=$ $U(g)^{-1} f(g x)$, where $g x$ is in $\Omega$. To see that $E f$ is well behaved, let $E(g) f$ be the function on $g^{-1} \Omega$ given by $E(g) f(x)=U(g)^{-1} f(g x)$. Condition (2.2) is equivalent to

$$
\left(U_{i \sigma}-(-1)^{\alpha_{i}} I\right) D^{\alpha} f=0 .
$$

This condition can be used to show that $D^{\alpha} f$ and $D^{\alpha} E\left(R_{i \sigma}\right) f$ agree on $(\partial \Omega)_{i \sigma}$, which implies that $D^{\alpha} E\left(g_{1}\right) f$ and $D^{\alpha} E\left(g_{2}\right) f$ agree on the intersection of their domains. Therefore $E f$ is well defined and $C^{\infty}$, and $E$ defines a map from $H_{V}^{\infty}\left(\Omega, C^{m}\right)$ to $H_{U}^{\infty}\left(R^{n}, C^{m}\right)$. $E$ is bijective: the operation of restricting functions on $R^{n}$ to $\Omega$ defines an inverse of $E$ (use condition (4.2) again).

(2) Conditions (2.3) hold if and only if conditions (4.1) hold. (One might show that both sets of conditions are equivalent to $A^{\alpha} U_{i \sigma}=(-1)^{\alpha_{i}} U_{i \sigma} A^{\alpha}$.)

(3) Let $f$ be in $H_{V}^{\infty}\left(\Omega, C^{m}\right)$. Then $E A F=A E f$ on $\Omega$. The hypothesis about $A$ implies that $E A f$ and $A E f$ are both in $H_{U}^{\infty}\left(R^{n}, C^{m}\right)$, and so must be equal. 
LEMMA 2. Suppose $A$ maps $H_{U}^{\infty}\left(R^{n}, C^{m}\right)$ to itself. Then for each $f$ in $H_{U}^{\infty}\left(R^{n}, C^{m}\right)$, there is a unique $\tilde{f}$ in $C^{\infty}\left(R^{n} \times R, C^{m}\right)$ such that $\tilde{f}$ solves problem (2.1) and each $f(, t)$ is in $H_{U}^{\infty}\left(R^{n}, C^{m}\right)$.

ProOF. We let $T$ be the subgroup of $G$ generated by elements of the form $\left(R_{i+} R_{i-}\right)^{2}$. Since the $U_{i \sigma}$ commute and are square roots of the identity operator, $U$ is trivial on $T$, and so functions that are equivariant with respect to $U$ are invariant under $T$. (If $V_{i+}$ were equal to $V_{i-}$ for all $i$, we could have let $T$ be the subgroup of $G$ generated by elements of the form $R_{i+} R_{i-}$ and still have $U$ trivial on $T$.) Let $a_{i}$ be $4\left(a_{i+}-a_{i-}\right)$, which we suppose to be $\infty$ if the $a_{i}$ are not both finite. Then $\left(R_{i+} R_{i-}\right)^{2}$ is translation by $a_{i}$ in the $i$ th direction. A fundamental domain for $T$ is $\tilde{\Omega}=\tilde{\Omega}_{1} \times \cdots \times \Omega_{n}$, where $\tilde{\Omega}_{i}$ is the closure of the interval $\left(-a_{i} / 2, a_{i} / 2\right)$. Let $H_{T}^{\infty}\left(R^{n}, C^{m}\right)$ be the space of functions in $C^{\infty}\left(R^{n}, C^{m}\right)$ that are invariant under $T$ and are extensions of functions in $H^{\infty}\left(\tilde{\Omega}, C^{m}\right)$. Since $\tilde{\Omega}$ is a finite union of translates of $\Omega$, functions that are equivariant with respect to $U$ and are square-integrable on $\Omega$ are also square-integrable on $\tilde{\Omega}$. Therefore $H_{U}^{\dot{\infty}}\left(R^{n}, C^{m}\right)$ is contained in $H_{T}^{\infty}\left(R^{n}, C^{m}\right)$.

Fourier transforms will be functions on $\hat{\Omega}=\hat{\Omega}_{1} \times \cdots \times \hat{\Omega}_{n}$, where $\hat{\Omega}_{i}$ is the lattice $2 \pi a_{i}^{-1} Z$ if $a_{i}$ is finite and is $R$ if $a_{i}$ is infinite. If $f$ is in $H_{T}^{\infty}\left(R^{n}, C^{m}\right)$, let $F f$ be the function on $\hat{\Omega}$ given by

$$
F f(p)=(2 \pi)^{-n / 2} \int_{\hat{\Omega}} e^{-i p \cdot x} f(x) d x .
$$

$F$ defines a bijection from $H_{T}^{\infty}\left(R^{n}, C^{m}\right)$ to $\hat{H}_{T}^{\infty}\left(\hat{\Omega}, C^{m}\right)$, the space of $\mu$-equivalence classes of measurable functions from $\hat{\Omega}$ to $C^{m}$ such that for each $\alpha$, the function $p \rightarrow p^{\alpha} f(p)$ is square-integrable with respect to $\mu$. Here $\mu=\mu_{1} \times \cdots \times \mu_{n}$, where $\mu_{i}$ is $2 \pi a_{i}^{-1}$ times counting measure if $a_{i}$ is finite, and is Lebesgue measure if $a_{i}$ is infinite.

Let $\hat{A}$ denote the function on $\hat{\Omega}$ defined by $\hat{A}(p)=\sum A^{\alpha}(i p)^{\alpha}$, and also denote the operation of multiplication by $\hat{A}$. Then $A=F^{-1} \hat{A} F$ on $H_{T}^{\infty}\left(R^{n}, C^{m}\right)$, and we expect that the function defined by $\tilde{F}(, t)=F^{-1} e^{\hat{A} t} F f$ solves problem (2.1). $\left(\tilde{f}\right.$ is well defined because $e^{\hat{A} t}$ maps $\hat{H}_{T}^{\infty}\left(\hat{\Omega}, C^{m}\right)$ to itself. (Use the formal skewadjointness of $A$.)) And, indeed, a standard Sobolev-space argument shows that $\tilde{f}$ is a $C^{\infty}$ solution of problem (2.1).

The image of $H_{U}^{\infty}\left(R^{n}, C^{m}\right)$ under $F$ is $\hat{H}_{U}^{\infty}\left(\hat{\Omega}, C^{m}\right)$, the space of $f$ in $\hat{H}_{T}^{\infty}\left(\hat{\Omega}, C^{m}\right)$ such that

$$
f(O(g) p)=e^{i p \cdot g 0} U(g) f(p)
$$

for all $g$ and $p$. Equation (4.1) implies that

$$
\hat{A}(p) U(g)=U(g) \hat{A}(O(g) p),
$$

which, in turn, implies that

$$
e^{\hat{A}(p) t} U(g)=U(g) e^{\hat{A}(O(g) p) t} .
$$

This equation can be used to show that $e^{\hat{A} t}$ maps $\hat{H}_{U}^{\infty}\left(\hat{\Omega}, C^{m}\right)$ to itself. Therefore each $\tilde{f}(, t)$ is in $H_{U}^{\infty}\left(R^{n}, C^{m}\right)$.

A standard energy argument shows that $f$ is unique. 
ProOF OF ThEOREM 1. Use Lemmas 1 and 2 to show that the function defined by

$$
\tilde{f}(, t)=E^{-1} \tilde{E} f(, t)
$$

is as in Theorem 1.

\section{REFERENCES}

1. J. B. Keller, The scope of the image method, Comm. Pure Appl. Math. 6 (1953), 505-512.

2. F. J. Massey and J. B. Rauch, Differentiability of solutions to hyperbolic initial-boundary value problems, Trans. Amer. Math. Soc. 189 (1974), 303-318.

3. S. Osher, Initial-boundary value problems for hyperbolic systems in regions with corners. II, Trans. Amer. Math. Soc. 198 (1974), 155-175.

4. L. Sarason and J. A. Smoller, Geometrical optics and the corner problem, Arch. Rational Mech. Anal. 56 (1975), 34-69.

School of Mathematics, University of Minnesota, Minneapolis, Minnesota 55455

Current address: Department of Mathematical Sciences, George Mason University, Fairfax, Virginia 22030 